\title{
ON THE DIFFERENTIAL INVARIANTS OF A PLANE*
}

BY

\section{CHARLES NELSON HASKINS}

The object of this note is the discussion of certain results recently obtained by Forsyth $\dagger$ and RICCI conceraing the differential invariants of plane curves.

\section{§1. Forsyth's † results.}

FonsYTH considers the differential form of class zero,

$$
d s^{2}=d x^{2}+d y^{2}=a d u^{2}+2 h d u d v+b d v^{2} ;
$$

one or more curves $\phi(u, v)=$ const., their radii of curvature $\rho$, the normal derivatives $d \phi / d u=C$, and the derivatives of $\rho$ and $C$ along the are and along the normal.

He obtains the following results :

(1) "There are $p^{2}$ absolute invariants which involve derivatives of $\phi$ up to order $p$ and are algebraically independent of one another."

(2) The quantities $C$ and $\rho$, with their derivatives up to orders $p-1$ and $p-2$ respectively, form a system of $p^{2}$ geometrical magnitudes of orders not exceeding $p$, "being exactly the same in number as the aggregate of algebraically independent invariants up to the same order. Hence all the geometrical magnitudes are independent of one another, and therefore there are no relations among the geometrical magnitudes," (i. e.) "among the quantities $C, \rho$, and their derivatives common to all curves in the plane. This result differs from the geometry of curves upon a curved surface and from the geometry of surfaces in space."

\section{§2. Ricci's results and their consequences.}

RICCI $\ddagger$ has proved theorems which may be stated as follows:

(1) Quadratic differential forms of class zero have no Gaussian differential invariants.

(2) The differential parameters of quadratic differential forms of class zero are the simultaneous algebraic invariants of the given forms and the linear,

\footnotetext{
* Presented to the Society April 28, 1906. Received for publication Angust 25, 1906.

†Forsyth, Rendiconti del Circolo Matematico di Palermo, vol. 21 (1906), pp. 115-125.

$\ddagger$ RICCI, Annali di Matematica, ser. 2, vol. 14 (1885), p. 11.
} 
quadratic, $\cdots, p$-ic forms whose coefficients are the first, second, $\cdots, p$-th covariant derivatives of $\phi$.

It therefore follows from Ricci's results that:

(1) There are

$$
3+2+3+\cdots+(p+1)-4=\frac{p^{2}+3 p-2}{2}
$$

absolute invariants which involve derivatives of $\phi$ of order up to $p$ and are algebraically independent of one another.

(2) There are

$$
R=p^{2}-\frac{p^{2}+3 p-2}{2}=\frac{(p-1)(p-2)}{2}
$$

relations among the $\rho^{2}$ geometrical magnitudes $C, \rho$, and their derivatives of orders not exceeding $p$.

\section{§ 3. Discussion of the results.}

The results of ForsyTh are obtained by consideration of the complete system of linear partial differential equations satisfied by the invariants in question - a method depending on the use of the infinitesimal transformations of the intinite group by which the form $d s^{2}$ is transformed.

Ricci's results are found by his method of the "Absolute Differential Calculus."

The two sets of results are contradictory except when $p=1$, or $p=2$. The reason for this is as follows. The number of invariants given by ForsYTH is obtained by subtracting from the number of independent variables in his complete system the number of equations comprising that system. Among these variables are the coefficients $a, h, b$, and their derivatives of various orders with respect to $u$ and $v$. Were the form of class unity, that is, reducible to $d x^{2}+d y^{2}+d z^{2}$ but not to $d x^{2}+d y^{2}$, these variables would all be independent, and the number of invariants would be $p^{2}$, a result included in those given by Zorawskr.* The form here under consideration is, however, of class zero ; that is, reducible to $d x^{2}+d y^{2}$. Consequently the four-, five-, etc., index symbols of Cirris'roffer. vanish identically, so that the derivatives of the coefficients $a, h, b$ are no longer independent, but are bound by relations. These are

$$
\begin{gathered}
(12,12)=0, \\
(12,121)=0, \\
(12,122)=0,
\end{gathered}
$$

* ZokAWsi, Acta Mathematica, vol. 16 (1892), p. 41. 


$$
\begin{aligned}
& (12,1211)=0, \\
& (12,1212)=0, \\
& (12,1222)=0,
\end{aligned}
$$

etc.

In the case of invariants of order $p>2$ there are

$$
R=1+2+3+\cdots+(p-2)=\frac{(p-1)(p-2)}{2}
$$

such relations, and hence only

$$
p^{2}-R=\frac{p^{2}+3 p-2}{2}
$$

independent absolute invariants.

The geometrical magnitudes $C, \rho$, and their derivatives specified in $(2), \S 1$, are absolute invariants, $p^{2}$ in number. This number is greater by $R$ than the number of independent absolute invariants of order $p$. Hence there must exist $R$ relations among these geometrical magnitudes. The lowest order for which $R \neq 0$ is $p=3$; for which $R=1$. Hence we should expect to find one relation among the geometrical magnitudes of the third order. It is easy to verify directly that the relation

$$
\frac{1}{C} \frac{d^{2} C}{d s^{2}}=\frac{1}{\rho^{2}}-\frac{d}{d n}\left(\frac{1}{\rho}\right)+2\left(\frac{1}{C} \frac{d C}{d s}\right)^{2}
$$

is satisfied by the magnitudes in question. Moreover, Fonsyth $\uparrow$ has already given, for curves on a surface, a relation among the geometrical magnitudes of the third order which reduces to this when the surface reduces to a plane.

MARION, MASS., August 22, 1906.

† Forsyth, Philosophical Transactions, ser. A, vol 201 (1902), p. 398. 